\title{
Uma Análise da Interação Humano-Computador da Inteligência Artificial do Google Fotos
}

\author{
Pedro Augusto Salamoni \\ Instituto de Computação, UFMT \\ Cuiabá, MT \\ salamonip@gmail.com.br
}

\author{
Vinícius Ferreira Galvão \\ Instituto de Computação, UFMT \\ Cuiabá, MT \\ vinifgalvao1@hotmail.com
}

\author{
Cristiano Maciel \\ Instituto de Computação, UFMT \\ Cuiabá, MT \\ crismac@gmail.com
}

\section{RESUMO}

Alguns aspectos como aumento de dados multimidia, expansão em compartilhamento, cultura de armazenamento e aumento da interação tecnológica contribuem para alguns desafios tecnológicos. Este artigo descreve as funcionalidades de Inteligência Artificial, desenvolvidos pelo Google Fotos, mostrando seus recursos, apresentando seu layout e executando testes com especialistas e com usuários de diferentes níveis de conhecimento sobre este software a fim de capturar suas reações e percepções sobre a aplicação.

\section{PALAVRAS-CHAVE}

Google Fotos, Experiência de Usuário, Avaliação de Comunicabilidade, Avaliação Heurística, Emocards

\section{INTRODUÇÃO}

Com o crescente volume de informações, se torna pertinente organizar massivos volumes de dados, o que é uma tarefa difícil. Neste sentido, para auxiliar no gerenciamento de imagens pessoais, em 2018, Google lançou o Google Fotos, mais de 5 bilhões de fotos são vistas todos os dias através do aplicativo [9]. Ele atingiu 3.72 petabytes de fotos e vídeos em 2017 [5]. Além do seu sucesso, a plataforma continuou crescendo durante os anos, chegando a 500 milhões de usuários com uploads de 1.2 bilhões de fotos todos os dias [10]. Assumindo a dificuldade em compartilhar e organizar todas elas, a ferramenta faz uso da Inteligência Artificial (IA) e disponibiliza a usuários diversas funcionalidades, de forma fácil e rápida. Visto isso, é pertinente avaliar a forma que esta ferramenta utiliza IA para interagir com seus usuários. Neste estudo, optou-se pelo uso de diferentes estratégias da área de Interação Humano-Computador (IHC): Avaliação Heurística

Permission to reproduce or distribute, in whole or in part, material extracted from this work, verbatim, adapted or remixed, as well as the creation or production from the content of such work, is granted without fee for noncommercial use, provided that the original work is properly credited.

IHC 2019 - TRILHA PÔSTERES E DEMONSTRAÇÕES, Outubro 21-25, 2019, Vitória, Brasil. In Anais Estendidos do XVIII Simpósio Brasileiro sobre Fatores Humanos em Sistemas Computacionais. Porto Alegre: SBC.

(C) 2019 by the author(s), in accordance with the terms of the Creative Commons Attribution-NonCommercial 4.0 International Public License (CC BY-NC 4.0).
(AH), a fim de inspecionar as funcionalidades da aplicação, o Teste de Usabilidade (TU), para observar o uso da ferramenta com voluntários e, finalmente, a mensuração da experiência de usuários (UX) via Emocards. Por meio destas, este artigo objetiva inspecionar a usabilidade e comunicabilidade dos elementos de IA do Google Fotos e, por meio dos resultados, refletir sobre seu papel.

\section{GOOGLE FOTOS}

O Google Fotos é o sucessor dos recursos de fotográficos incorporados no Google+ [7]. A ferramenta oferece armazenamento de fotos e ferramentas organizacionais. O serviço mudou sua associação de uma plataforma de compartilhamento para uma plataforma de biblioteca privada. Diferentes formas de Machine Learning [4, 8] no software permitem o reconhecimento do conteúdo das fotos, geram álbuns automaticamente, animam fotos semelhantes em vídeos rápidos, armazenam memórias passadas em momentos significativos e melhoram a qualidade de fotos e vídeos [2].

\section{METODOLOGIA}

A fim de investigar a IA da ferramenta serão detalhados os métodos utilizados. Para a $\mathrm{AH}$, foram requisitados que 3 avaliadores experientes em IHC realizassem os testes em conjunto. No Teste de Usabilidade e Emocards foram selecionados 3 voluntários com pouco ou nenhum conhecimento da ferramenta. Todos participantes assinaram um termo de consentimento para permissão da gravação, registro e observação durante os testes.

A AH compreende um método para verificação de uma lista de regras Heurísticas [3], útil para medir possíveis problemas de interação com usuário em qualquer etapa de desenvolvimento do software. A AH realizada pelos 3 especialistas seguiu o roteiro: preparação dos testes, incluindo a construção do ambiente do Google Fotos, com fotos que possuam características para serem avaliadas; acompanhamento dos procedimentos na execução do teste em tarefas corriqueiras ao usuário; consolidação da lista de problemas anotados; e análise dos problemas encontrados segundo o grau de severidade, documentação e apresentação dos resultados.

O objetivo principal do TU é avaliar a qualidade de um sistema com relação a fatores que os projetistas definem 
como sendo prioritários ao sistema [6]. Para o TU, foram selecionados voluntários que tivessem pouca ou nenhuma experiência no software. Para coleta de suas opiniões sobre o software, eles foram dispostos em uma sala com pouca interferência externa, sendo analisados pelo avaliador [6].

Os testes tiveram natureza qualitativa, sendo assim cada voluntário teve a mesma influência no resultado final. Para a realização do TU, os voluntários aderiram ao seguinte roteiro: foi pedido ao voluntário importar e lembrar-se de detalhes contidas em suas fotos para identificação; após isto, ele/ela deveria escrever numa folha de papel os detalhes a serem pesquisados, para verificação na plataforma; foi requerido que fizessem uma busca de um objeto e pessoas específicas contidas em suas fotos na IA do Google Fotos; e o avaliador preencheu um formulário com o relato dos problemas e/ou dificuldades enfrentadas durante TU.

O Emocards é uma das técnicas projetadas para medir as respostas emocionais do usuário e sua experiência com o software. Ela é baseada no modelo emocional definido por Russel, contendo 8 emoções principais consideradas para análise de expressões faciais [1]. Conforme o TU avançasse, os participantes e suas reações emocionais foram observadas. Os voluntários tiveram sua UX registradas sem nenhum conhecimento prévio, assim obtendo reações autênticas durante o TU. As reações foram registradas em 2 situações diferentes enfrentadas pelo voluntário: a descoberta da IA de busca de imagens da ferramenta; e durante o processo de funcionamento da IA e visualização dos resultados.

\section{RESULTADOS}

Os especialistas concluíram que foram encontrados problemas de usabilidade via $\mathrm{AH}$ [3] classificados como: Ajuda e Documentação - "dificuldade na intuição da disponibilidade de pesquisa de objetos pela IA" deixando o usuário limitado nas formas de interação; Flexibilidade e Eficiência de Uso "dificuldade de redefinir o item buscado pela IA" deixando o usuário perdido sobre quais ações realizar para redefinição do mesmo; e Visibilidade do status do sistema - "fraca identificação dos rostos das pessoas, gerando links errôneos" deixando o usuário desacreditado sobre eficiência da classificação.

Assim como a AH, o TU foi guiado por especialistas, observando os usuários de dois níveis de conhecimento diferentes. Depois de alguns minutos sem interferência, e após recolhimento dos formulários, os avaliadores levantaram as seguintes observações: alto tempo de execução da IA ao procurar itens inexistentes, sendo que o usuário espera um grande tempo até que se recuse a busca; demora no reconhecimento facial inicial nas mídias importadas; item de difícil reconhecimento em identificar características pertencentes ao local (cachoeiras, parques)

No teste de Emocards, para cada voluntário foram registradas destintas emoções após o TU. Durante a descoberta e uso da IA, os voluntários 01 e 03, obtiveram experiência prazerosa no decorrer do teste, enquanto o voluntário 02 , obteve resultado neutro e calmo na descoberta da IA.

\section{CONCLUSÃO}

Neste estudo, foram identificados problemas durante os testes de interação em todas avaliações. Todavia, das dez avaliações heurísticas possíveis, apenas em três foram percebidos problemas pelos especialistas. As avaliações com usuários mostraram que, apesar do relato de problemas de comunicação descontinuada, a experiência deles foi positiva, tendo em vista as emoções percebidas e o atingimento dos seus objetivos com facilidade.

Por fim, a IA se apresentou satisfatória em cumprir o proposto, contudo, com ressalva dos pontos apresentados acima. Todavia, é um desafio realizar testes de IHC que envolvem IA, tema que merece ser mais bem pesquisado em estudos preliminares como esse. A pesquisa ainda deve ser expandida por meio de buscas em objetos mais complexos, bem como utilizando fotos/imagens de difíceis reconhecimento pela IA.

\section{REFERÊNCIAS}

[1] Anshu Agarwal and Andrew Meyer. 2009. Beyond Usability: Evaluating Emotional Response As an Integral Part of the User Experience. In CHI '09 Extended Abstracts on Human Factors in Computing Systems (CHI EA '09). ACM, New York, NY, USA, 2919-2930. https://doi.org/10.1145/1520340.1520420

[2] Richard Gao. 2017. Google Photos' video stabilization gets demoed, proves impressive. (2017). https://www.androidpolice.com/2017/04/13/ google-photos-video-stabilization-gets-demoed-proves-impressive/

[3] Jakob Nielsen. 1995. How to conduct a heuristic evaluation. Nielsen Norman Group 1 (1995), 1-8.

[4] Paul Nieuwenhuysen. 2018. Information Discovery and Images A Case Study of Google Photos. In 2018 5th International Symposium on Emerging Trends and Technologies in Libraries and Information Services (ETTLIS). 16-21. https://doi.org/10.1109/ETTLIS.2018.8485238

[5] Chris Perry. 2015. 11 things to know about Google Photos. (2015). https://blog.google/products/photos/11-things-to-knowabout-google-photos/

[6] Raquel Oliveira Prates and Simone Diniz Junqueira Barbosa. 2003. Avaliação de interfaces de usuário-conceitos e métodos. In fornada de Atualização em Informática do Congresso da Sociedade Brasileira de Computação, Capítulo, Vol. 6. 28.

[7] Anil Sabharwal. 2016. Moving on from Picasa. (2016). http: //googlephotos.blogspot.com/2016/02/moving-on-from-picasa.html

[8] Kai-En Tsay, Yi-Leh Wu, Maw-Kae Hor, and Cheng-Yuan Tang. 2009. Personal Photo Organizer Based on Automated Annotation Framework. In 2009 Fifth International Conference on Intelligent Information Hiding and Multimedia Signal Processing. 507-510. https: //doi.org/10.1109/IIH-MSP.2009.165

[9] Steven Winkelman and Daven Mathies. 2018. Google Photos now using A.I. to simplify editing and sharing images. (2018). https://www. digitaltrends.com/photography/google-photos-suggested-edits/

[10] Ken Yeung. 2017. Google Photos passes 500 million users, gets better sharing features and $\$ 10$ photo books. (2017). https://venturebeat. com/2017/05/17/google-photos-passes-500-million-users/ 